\title{
Application of spectral analysis to heart sounds analysis
}

\author{
XuKunLiang ${ }^{1, a}$ \\ ${ }^{1}$ QuJing Normal University Computer science and Engineering, YunNan QuJing 655011,China \\ a422078334@qq.com
}

Keywords: Gas, A burglar alarm, Single chip microcomputer

\begin{abstract}
At first, this paper analyzes the family use combustible gases present situation and the existence risk, then atc89c52 single chip microcomputer based on, using sensor mq-2 gas concentration detection, to collected the gas concentration data as the basis for the control of sound, alarm device, to achieve the function of safety warning, for household gas safety has certain use value.
\end{abstract}

\section{Introduction}

In daily life, most of the families in the use of gas, with the improvement of living standard, the family kitchen design is more delicate, usually to hide gas bottle, pipeline design and decoration, such as the pipeline, liquefied gas bottle hidden hearth ark inside, once the gas leak, can make the gas quickly gathered in a narrow space, higher concentration, it is easy to cause the occurrence of explosion and fire [1]. Therefore, in the family to use gas leak detection and alarm prompt appears very necessary, this paper designed a single-chip microcomputer as the core, with peripheral circuit to signal acquisition and voice alarm alarm, by default gas concentration, if leaking gas concentration exceeds the preset concentration, alarm system will alarm, improve the safety of the gas, at the same time, the alarm low cost [2].

\section{The project design}

In the design, single-chip computer is the center of the design, on the one hand, receiving sensor detects the combustible gas concentration of the analog voltage signal, on the other hand to the signal processing, control alarm circuit, control circuit for the corresponding operation. According to the functional requirements, mainly include sensor circuit, single chip microcomputer interface to the hardware circuit, voice alarm control circuit three parts [3-5], specific system structure are shown in figure 1 below.

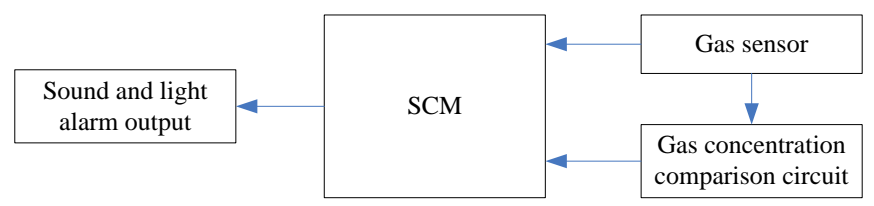

Fig.1 home combustible gas alarm system structure

\section{The hardware design}

(1) gas sensor

MQ - 2 gas sensor used by the gas sensitive material is in clean air conductivity low tin oxide ( $\mathrm{SnO} 2)$. When sensor exist combustible gas in the environment, the sensor of electrical conductivity increases with the increased concentration of combustible gas in the air. Using a simple circuit can converts the change of the conductivity and the gas concentration corresponding to the output signal. MQ - 2 gas sensor sensitivity of liquefied petroleum gas, propane, hydrogen gas and other combustible vapor detection is ideal. The sensors can detect a variety of combustible gas, is a low-cost sensor suitable for many applications [6]. The main features and application: in a wider range of concentration of combustible gas with good sensitivity; High sensitivity of liquefied petroleum gas, propane, hydrogen; Long life, low cost; Simple drive circuit; Family with gas 
leakage alarm; Industrial combustible gas alarm; Portable gas detector. Suitable for use in the household gas concentration detection, sensor simulation circuit is shown in figure 2.

(2) A/D conversion circuit

ADC0832 is one of the national semiconductor production 8-bit resolution, dual channel A/D conversion chip. Can meet the requirements for general analog conversion [8]. The chip simulation between $0 \sim 5 \mathrm{v}$ voltage input. Conversion time is $32 \mathrm{mu} \mathrm{S}$, fast converting speed and stable performance. Chip with independent can make input, through DI data input, can easily realize the choice of channel function [8]. A/D conversion circuit simulation is shown in figure 2.

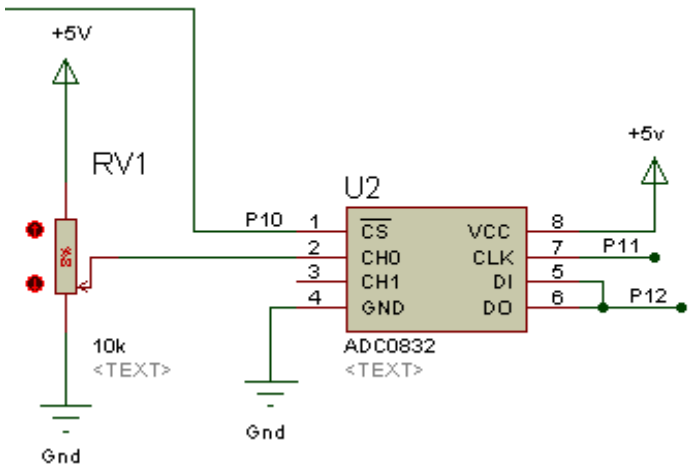

(3) the sound and light alarm

Fig.2 sensors, A/D conversion circuit simulation

When the concentration of combustible gas exceeds limit, speakers would call the police. The simulation circuit as shown in figure 3, composed of transistor and speakers. When the actual detection concentration is lower than the set concentration, triode conduction, the speaker doesn't work. When the concentration of the actual detection concentration equal to or more than set, through P2.2 connected with MCU that cause the change of the level, P2.2 as low level, triode conduction, the speaker alarm sounds.

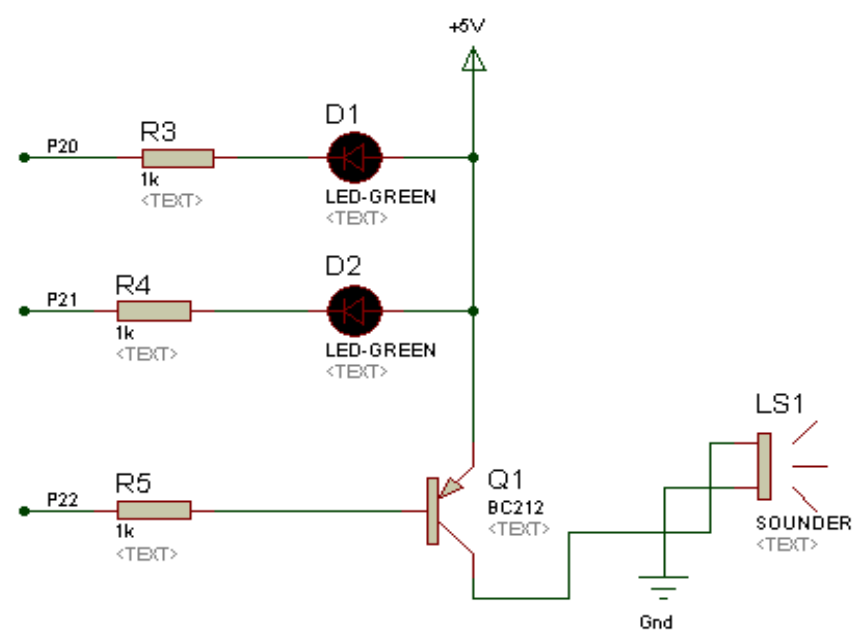

Fig.3 sound and light alarm circuit

(4) microcontroller interface circuit

Choose play machine is STC89C52, this article is on STC company production of a low power consumption, high performance CMOS8 microcontrollers, with $8 \mathrm{k}$ in system programmable Flash memory. Design of simulation circuit diagram as shown in figure 4. 


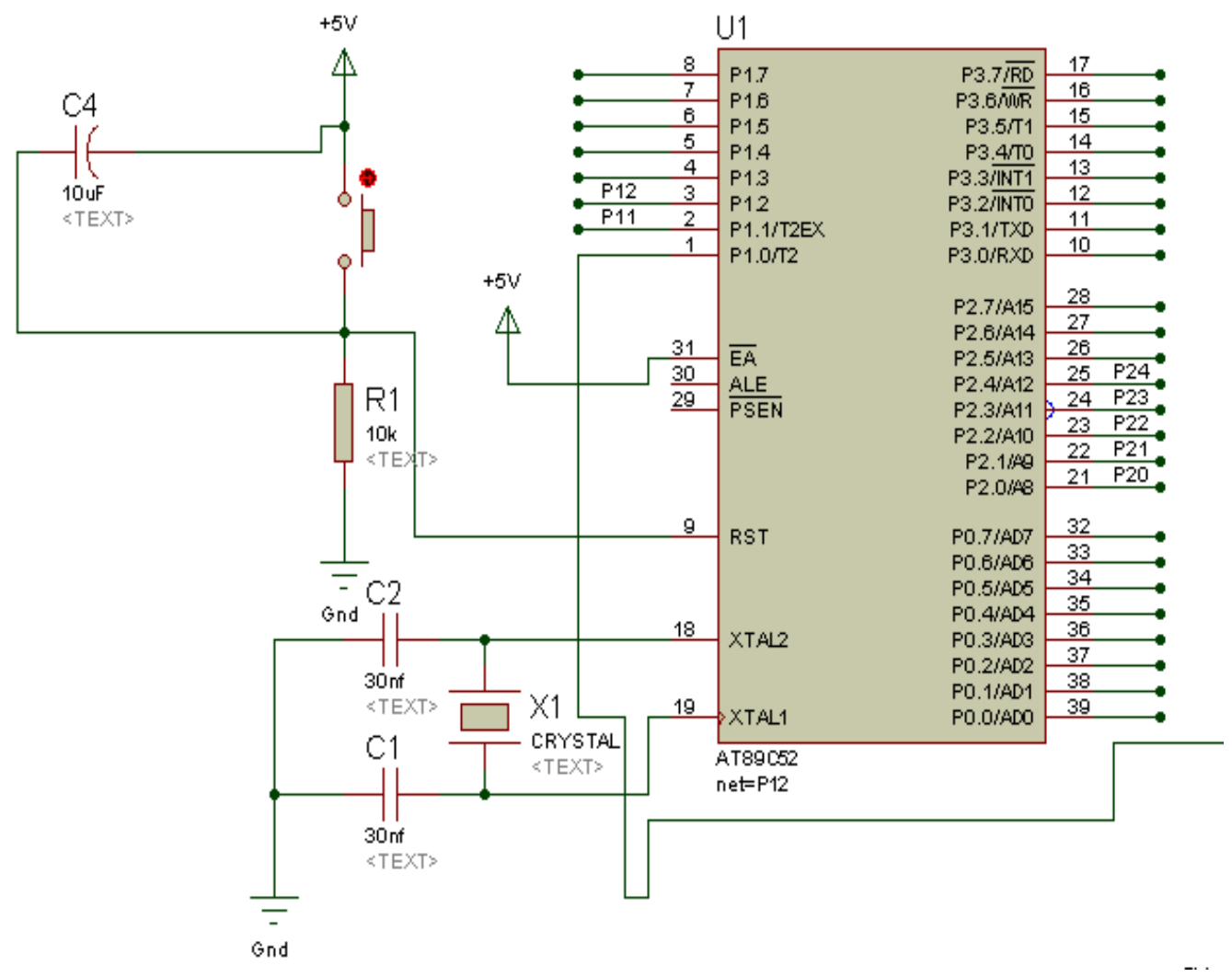

Fig.4 family combustible gas alarm single-chip microcomputer simulation circuit diagram

\section{The software design}

Main right main program, alarm, alarm subroutine of $\mathrm{A} / \mathrm{D}$ conversion program. Alarm main program flow chart is shown in figure 5.

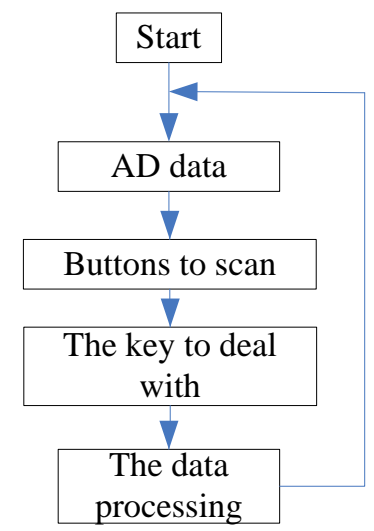

Fig.5 main program flow chart

\section{Conclusion}

Home combustible gas, the author of this paper the problems existing in the use process, designed a combustible gas detection alarm, in the system using the MQ - 2 gas sensor detecting gas concentration. With the single chip processor as the core to control the alarm. Through simulation experiment, when the concentration of gas concentration than setting, alarm immediately make alarm prompt. To improve the safety of gas use, can satisfy the accuracy of the practical application requirements. 


\section{Reference}

[1]C.X.Li,The analysis of civil liquefied gas leak explosive power,Fire science and technology,Vol.23,No.2, pp. 173-174,2004.

[2]X.Y.Chen,Technology on the combustible gas detection alarm,Zhejiang: zhejiang university of technology,Master's thesis,2011.

[3]Z.H.Hu,H.N.Li, Gas alarm circuit design based on single chip microcomputer,Private science and technology,No.7,pp.73,2015.

[4]Q.H.Sun,L.Y.Zhang,Y.Han,Intelligent combustible gas leak detection alarm system design and implementation,Hebei industrial science and technology,Vol.25,No.1,pp.32-38,2008.

[5]L.C.Gao,T.Liang,Light ionization gas concentration detection based on C8051f040 singlechip circuit design,Electronic devices,Vol.3,No.38,pp.632-635,2015.

[6]H.W.Cang,The research and application of gas sensor array detection system,Xian: Xian university of electronic science and technology,Master's thesis, 2000.

[7]F.C.Jiang,C.P.Zhu,The present situation of the gas concentration detection technology and application,Journal of hohai university, changzhou campus, Vol.1,No.18,pp.16-19,2004.

[8]Information

on:http://wenku.baidu.com/link?url=Z9I_ltuGtujF2fGxT18QRABoTyzjGzs7jcNbIL19UNc7uxdaG6 czUaCb-C1tiRYW9Ry81rbdTDCkMjGX4p07hN5oB2GXnqYsEGIrReI1X3C 